\title{
THE USE AND UNDERSTANDING OF THE PROXIMATE STATUS INDICATION IN TRAFFIC DISPLAYS
}

\author{
Michael Zuschlag, Divya Chandra, Rebecca Grayhem \\ Volpe National Transportation Systems Center, Cambridge, MA
}

\begin{abstract}
Traffic Collision Avoidance System (TCAS) traffic displays use symbol fill to distinguish "proximate" from "non-proximate" targets, where proximate targets are within a specified range and altitude of ownship, and non-proximate targets are outside these parameters. While this is satisfactory for TCAS displays, Cockpit Displays of Traffic Information (CDTI) can present much more information than TCAS displays, and an alternative use of symbol fill may be preferred. Since a symbol for traffic has a limited number of visual features for encoding information, CDTI symbology should only encode information that can be used effectively by pilots. This study evaluated the utility of the proximate status indication in an effort to understand whether it is useful enough to show on CDTIs.
\end{abstract}

One hundred and one corporate and airline pilots were recruited for the web-based study via advertisements in on-line pilot newsletters. Pilots viewed videos of traffic scenarios depicted on a traffic display. Half the pilots were shown displays with a proximate status indication, and half the subjects were shown displays without a proximate status indication. Results showed that the proximate status indication did not improve consistency of pilot ratings relative to objective measures of threat and visibility. Furthermore, pilots who had the proximate status indication were $9 \%$ less accurate on average in selecting the highest threat traffic, whether the most threatening traffic was proximate or not.

Pilots also answered questions about the proximate indication status in TCAS. Most pilots reported they found it useful, usually for prioritizing their attention. Most pilots recognized that a proximate symbol must be within a specific range and altitude, but nearly equal proportions of pilots also believed proximate traffic must always be a higher threat than non-proximate traffic, which is not the case.

The combined results suggest that when assessing traffic threat, pilots place greater weight on the closeness of traffic than other key characteristics such as relative closing speed.

\section{Background and Motivation}

Many airline and corporate pilots have experience with the Traffic Alert and Collision Avoidance System (TCAS). In the future, TCAS traffic displays may be integrated with Cockpit Displays of Traffic Information (CDTIs) that obtain information about other aircraft from Automatic Dependent Surveillance-Broadcast (ADS-B) and other Aircraft Surveillance Applications Systems (ASAS). CDTIs will have the ability to show information about other aircraft that is not available to TCAS.

During the development of the recently published Minimum Operational Performance Standards (MOPS) for Aircraft Surveillance Applications System (ASAS) [1], there was discussion of whether CDTI symbols would be required to match TCAS traffic symbols in certain ways. On one hand, it is desirable to retain aspects of TCAS symbology because of extensive pilot experience with TCAS. On the other hand, there are only so many visual features that symbology can use to encode information before pilots start to become confused [2]. It is difficult to create a symbol set for an integrated display that graphically encodes all information found in both CDTI and TCAS displays. However, the difficulty may be reduced by the symbology only encoding information that is used effectively by the pilot.

\section{Proximate Status Indication}

TCAS traffic displays use symbol fill to distinguish "proximate" and "non-proximate" targets, where proximate targets are within a specified range and altitude of ownship (see Table 1).

CDTI manufacturers would like more flexibility to use symbol fill for other purposes. Questions have been raised about the value of the proximate status 
indication in general, its value relative to other potential traffic information, and about whether there are alternate ways of depicting target proximity, other than with symbol fill.

Table 1. TCAS Proximate Status Indication

\begin{tabular}{|l|c|l|}
\hline Status & Symbol & Criteria \\
\hline Proximate & $\diamond$ & $\begin{array}{l}\text { Closer than 6 nm laterally } \\
\text { and } 1200 \mathrm{ft} \text { vertically }\end{array}$ \\
\hline $\begin{array}{l}\text { Non- } \\
\text { proximate }\end{array}$ & $\diamond$ & $\begin{array}{l}\text { Farther than } 6 \mathrm{~nm} \text { laterally or } \\
1200 \mathrm{ft} \text { vertically }\end{array}$ \\
\hline
\end{tabular}

The purpose of this study was to assess the utility of the proximate status indication on a traffic display and to explore the implications for CDTI. Results of this study are expected to be considered by the Federal Aviation Administration (FAA) in the development of a Technical Standard Order (TSO) that may address the display of a proximate status indication in future CDTIs, including those that are integrated with TCAS. Because TCAS does not contend with tight competition among information for symbol features, the results are not necessarily relevant for TCAS-only displays.

\section{Potential Functions}

While the TCAS standards [3] describe the intended function of the traffic display and require that proximate traffic be displayed during alerts, they do not explicitly document the intended function of the proximate status indication (the use of fill) in terms of how it is expected to affect pilot performance. Whatever the original intent for the proximate status indication, we are interested in the potential uses pilots have developed for the proximate status indication that affect the operational use of TCAS. However, it appears that no previous research has investigated what these uses might be and how effective the proximate status indication is in supporting them.

Informal conversations with pilots suggest that the proximate status indication may be useful for estimating the threat level of traffic that is not in an alert status. More specifically, the proximate status indication may assist pilots to focus their visual search in advance on traffic that is likely to become a Traffic Advisory (TA). Regarding filled symbols as potential threats is not entirely consistent with TCAS logic, but it is nonetheless understandable and possibly useful. While the criteria for a TA depends primarily on time until the point of closest approach (CPA) [4] rather than proximity or distance to the traffic, closer traffic is in general more likely to become a TA than more distant traffic.

Another possibility is that the proximate status indication aids in deciding whether to attempt visual acquisition. Traffic more than $6 \mathrm{~nm}$ away is unlikely to be visible [5], so the proximate status indication might cue pilots to attempt visual search.

Using the proximate status indication for assessing threats or potential for visual acquisition are both consistent with the intended function of the TCAS traffic display [3]. Our study also sought to discover other uses of the proximate status as well.

\section{Research Questions}

To assess the utility of the proximate status indication our study attempts to:

- Experimentally measure the degree to which the proximate status indication potentially aids the pilot assessment of traffic threat and visibility.

- Experimentally measure the degree to which the proximate status indication potentially interferes with the pilot assessment of traffic threat.

- Gather pilots' subjective operational experiences with the proximate status indication; what it is used for, and how it may aid or interfere with traffic-related activities.

- Assess pilot knowledge and understanding of the proximate status indication.

The last item is of interest because pilot knowledge is another indicator of the current value of the proximate status indication. Operationally TCAS appears to be a success. So, if TCAS-experienced pilots are using TCAS successfully without knowing the actual meaning of the proximate status indication, then it could be argued that the proximate status indication is not strictly necessary.

\section{Method}

The study was completed with web-based materials for quick results at a reasonable cost. 


\section{Participants}

Advertisements were placed in newsletters for the Air Line Pilots Association (ALPA) and the National Business Aviation Association (NBAA) to recruit airline and corporate pilots based in the United States. The announcements asked pilots with TCAS experience to visit a website in order to complete the study.

The participants answered questions about their background on one of the first pages. The site allowed only participants who indicated the appropriate pilot rating or certification and TCAS experience to complete the study, as the results were intended to generalize to pilots with TCAS experience. Because it was a web study, there was no way to independently verify whether each participant was actually a pilot.

There were 136 participants of whom 101 completed all tasks in the study, yielding a $31 \%$ attrition rate, which is comparable to a previous CDTI web study [2]. One hundred and ten completed the Traffic Rating task, 102 completed the Greatest Threat task, and 101 completed the remaining tasks.

Table 2 shows a breakdown of the participants by type of operations they conduct, experience with TCAS, and average flight hours. In general, experience was high. Most had air transport experience and many had corporate experience. All participants reported some TCAS experience, typically with TCAS II.

Table 2. Participant characteristics.

\begin{tabular}{|l|c|c|c|c|}
\hline \multirow{2}{*}{ Operation } & \multirow{2}{*}{$\begin{array}{c}\text { Flight } \\
\text { Hours } \\
\text { (avg) }\end{array}$} & \multicolumn{2}{|c|}{$\begin{array}{c}\text { Experience } \\
\text { (number of pilots) }\end{array}$} & \multirow{2}{*}{$\begin{array}{c}\text { Total } \\
\text { (\# of } \\
\text { pilots) }\end{array}$} \\
\cline { 3 - 4 } & TCAS I & TCAS II & TCir \\
Air & 8793 & 3 & 69 & 70 \\
\hline Transport & & & & \\
\hline Military & 12675 & 1 & 16 & 16 \\
\hline Private Only & 630 & 3 & 0 & 3 \\
\hline Overall & 8837 & 12 & 96 & 101 \\
\hline
\end{tabular}

\section{Procedure Overview}

Figure 1 illustrates the procedure for each participant. The study was divided into four tasks that the pilots completed sequentially:

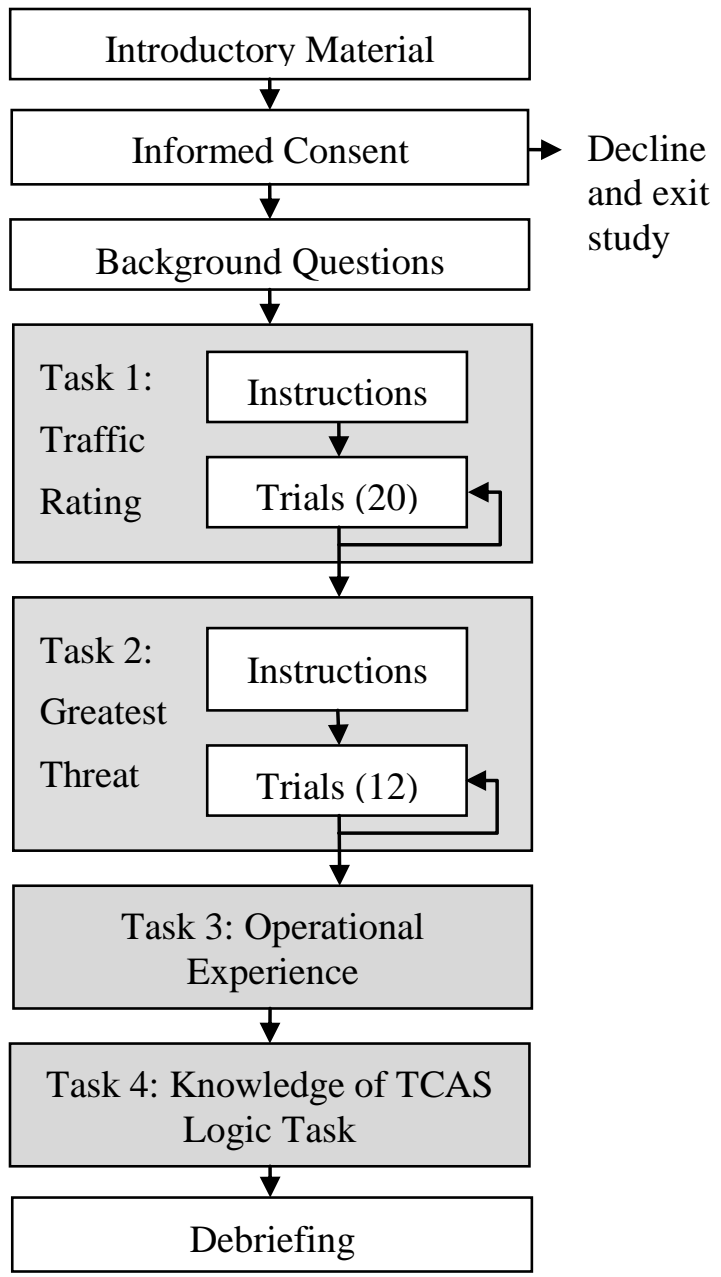

Figure 1. Procedure

- Task 1: Traffic Rating. This task evaluates the degree to which the proximate status indication may aid in threat or visibility assessment.

- Task 2: Greatest Threat. This task evaluates to what degree the proximate status indication may interfere with accurate threat assessments.

- Task 3: Operational Experience. This task contained open-ended questions about operational use of the proximate status indication.

- Task 4: Knowledge of TCAS Logic. This task consisted of a series of factual questions about the definition of the proximate status indication. 


\section{Task 1: Traffic Rating}

The purpose of the Traffic Rating task was to evaluate the capacity of the proximate status indication to facilitate pilot assessments of:

- Traffic threat level, in order to anticipate a potential TA, and

- Ease of visual search, in order to decide whether initiating a visual search is worth the effort.

This was accomplished by presenting 20 trials, each with a 15-second video where traffic threat and potential for visual acquisition were realistically correlated with the proximate status of the traffic. Figure 2 shows a screen capture from an example video.

When the video ended, all information in the display was removed except for the symbol of one experimenter-chosen traffic aircraft. In all cases, the chosen traffic was generally converging laterally and vertically on ownship. Pilots subjectively rated chosen traffic for threat and potential for visual acquisition on forced-choice scales (from 0 to 10).

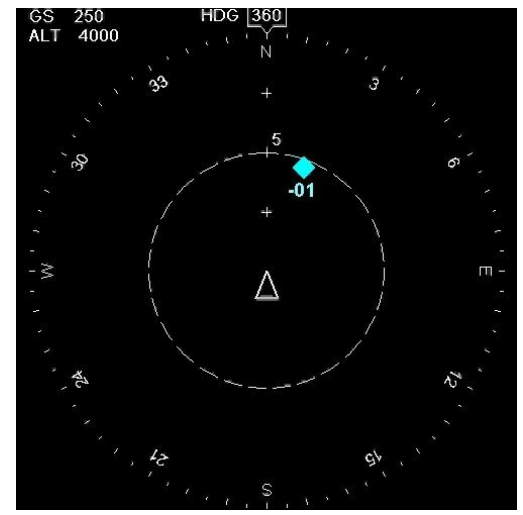

\section{Figure 2. Frame from a Traffic Rating video}

The chosen traffic varied in range and relative speed across the 20 trials. The net effect was that time to closest point of approach (CPA), which is the parameter that TCAS primarily uses to assess threat, varied across trials. The variability in range also represents variation in the objective visibility of the traffic, with more distant traffic being harder to see than closer traffic.

The ranges and speeds were chosen to be similar to that encountered operationally, based on an analysis of radar data from the New York terminal area. The correlation between the proximate status indication and time to CPA was actually a little higher in these test scenarios than in the radar data so the proximate status indication was a slightly better indication of threat in these videos than in reality. All targets also varied across the videos in their angles relative to the ownship and whether or not they were changing altitude. All aircraft held straight trajectories. Also, targets did not change from proximate to non-proximate status (or vice versa) during a single trial (15-second video).

The videos were refined through multiple iterations, reviews, and pilot testing to achieve a reasonable level of difficulty and realism.

\section{Experimental Conditions}

Each pilot was randomly assigned to one of four different conditions created by crossing the two independent variables, Proximate Status Indication and Traffic Density.

Proximate Status Indication had two levels:

- With Indication, where the display had filled symbols for proximate aircraft and unfilled symbols for non-proximate aircraft, as seen on TCAS displays.

- Without Indication, where the display had only unfilled symbols (i.e., symbols for proximate targets were identical to the symbols for non-proximate targets).

Instructions provided before the task listed all symbols the display would show and warned pilots that "the display does not have all the features of TCAS," so pilots would not be surprised or concerned by displays without the proximate status indication (i.e., filled symbols).

Traffic Density also had two levels:

- Low Density, with a single target within $10 \mathrm{~nm}$ that, of course, became the chosen target.

- High Density, with five targets within $10 \mathrm{~nm}$, which corresponds to the 80th percentile from the radar data from the New York terminal area.

The Traffic Density variable was included to allow comparisons between cases where pilots can devote all their attention to a single target and cases where pilots must divide their attention. In the latter situation, pilots may need to rely more on 
information that can be extracted quickly from a target symbol, for example, the information represented by symbol fill.

\section{Performance Measures}

To assess the potential for the proximate status indication to aid pilots, we compared pilots' threat ratings with the objective threat level as calculated by TCAS logic. TCAS logic was used as the standard for objective threat because:

- The study concerned the use of the proximate status indication specifically for anticipating TAs.

- The TCAS definition of threat is the result of extensive work by the expert aviation community, and has wide acceptance.

- The TCAS definition has proven operationally to perform excellently.

For the kinds of encounters presented in the videos, TCAS determines threat by the time to CPA and miss distance between target and ownship at CPA. Proximity (i.e., closeness) of the target enters into the threat calculation indirectly in that time until CPA depends on closeness and relative speed of closure.

To measure how much threat ratings correlated with actual threat, we performed a least-square multiple regression for each pilot of time to CPA, and vertical and horizontal distance at CPA on the pilot's 20 threat ratings. The result is a metric, $R^{2}$, which is a unit-less coefficient between 0 and 1 , that represents the consistency of each pilot's ratings with actual threat (i.e., the degree that traffic with higher time to CPA and greater miss distances are consistently rated as less threatening than traffic with lower time to CPA and smaller miss distances).

An $R^{2}$ of 0 indicates no relation between actual threat and the participants' ratings, suggesting either (a) pilots do not regard threat to be related to traffic closeness, relative speed, time to CPA, or miss distances, or (b) pilots are unable to reliably extract from the display the closeness, relative speed, time to CPA, or miss distances of traffic. For these particular videos, if pilots adopted a simple strategy of regarding any proximate traffic as threatening and any non-proximate traffic as non-threatening, ignoring relative speed, time to CPA, and miss distances, then the $R^{2}$ would be 0.18 . An $R^{2}$ of 1.0 would indicate that pilots can perfectly estimate relative time to $\mathrm{CPA}$ and miss distances from the videos.

Visibility ratings were evaluated through a similar process, where "actual" visibility is the combination of range and target position relative to the horizon (a "Ground" parameter), and the Rangeby-ground interaction. The Ground and Range-byground parameters accounted for the degree pilots include ground clutter effects in their traffic visibility assessments. The Ground parameter accounted for the potential of ground clutter to have a constant effect on visibility irrespective of range, while the Range-by-ground-interaction parameter accounted for the potential of visibility falling off at different rates with increasing range depending on whether or not the traffic appeared over ground clutter.

If the proximate status indication helps pilots quickly estimate distances, then it should produce more consistent threat and visibility assessments.

Performance is collectively indicated by the weights (the $B$ coefficients) for each pilot's regression equation. These can be used to discriminate certain cases where a pilot is consistent but not accurate. For example, with the threat rating, a pilot can completely ignore time to CPA and simply rate targets based only on miss distance. They can be consistent with this, resulting in a high $R^{2}$, but that is not a very complete mental model of threat.

The standardized $B$ s represent the weight a pilot puts on each component of threat. For example, maybe pilots with the proximate status indication attend more to miss distances than pilots without because it is easier for them to estimate range and therefore time to CPA. This would appear as a significant difference between the standardized $B$ s for the miss distances for pilots with and without the indication.

For this purpose we recomputed the regressions with time to CPA broken down into its components: relative speed and range. Of course, time is range divided by speed, not the weighted sum of range and speed, but with our particular experimental setup, time to CPA is accurately estimated by a weighted sum of standardized range and speed values (correlation $R=0.90$ ). This allows the analysis to indicate the emphasis pilots put on time, speed, and range in threat assessments. If pilots consider range and speed equally, then they are in effect looking at 
time to closest approach, and are mentally modeling threat as TCAS does. If pilots use range more than speed, then they are using range in addition to time, and vice versa if they use speed more than range. It is possible that for assessing threat the proximate status indication makes range seem more important to pilots than it is in TCAS.

\section{Task 2: Greatest Threat}

The purpose of the Greatest Threat task was to evaluate the potential for the proximate status indication to interfere with proper pilot assessment of traffic. This is in contrast to the Threat Rating task, which was designed to evaluate the potential for the proximate status indication to aid proper pilot assessment of traffic.

While traffic proximity is correlated with actual threat, it is not a perfect correlation; if there is sufficient difference in relative closing speed, farther traffic could be a higher threat, as defined in TCAS, than closer traffic. The Greatest Threat task presented pilots with cases where proximate traffic was more threatening than non-proximate traffic and vice versa in order to determine if pilots over-rely on the proximate status indication.

Pilots were shown 12 15-second videos of traffic displays. In every video there was always one proximate aircraft converging on the ownship, and one non-proximate aircraft converging on the ownship. At the end of each video, the context of the display was removed so that only the traffic and a letter next to each aircraft were visible to the participant. The participant then selected (by letter) the traffic they felt was most likely to produce a TA. The dependent variable for this task was whether participants accurately selected the aircraft that objectively had the greatest threat as determined by TCAS logic.

The greatest threat target, whether it was proximate or non-proximate, had a time to CPA of 49 seconds at the end of each video, and a 0 lateral and vertical miss distance at CPA. The lesser threat target had characteristics of one of the rows in Table 3 in each video. That is, the lesser threat targets varied in the way that they were a lesser threat, either because of a longer time to CPA or because they were on a track that would miss the ownship laterally or vertically.
Table 3. Lesser Threat Target Characteristics

\begin{tabular}{|c|c|c|c|}
\hline Target & Time to CPA & Lateral Miss & Vertical Miss \\
\hline 1 & $196 \mathrm{sec}$ & $0 \mathrm{~nm}$ & $0 \mathrm{ft}$ \\
\hline 2 & $49 \mathrm{sec}$ & $2 \mathrm{~nm}$ & $0 \mathrm{ft}$ \\
\hline 3 & $49 \mathrm{sec}$ & $0 \mathrm{~nm}$ & $1500 \mathrm{ft}$ \\
\hline
\end{tabular}

We chose these differences between the greater and lesser threats to achieve about a $75 \%$ chance, on average, of pilots being correct. The intent was to create a task that was sufficiently difficult so that pilots would use the proximate status indication. As in the Threat Rating task, all targets varied across the videos in their angles relative to the ownship and whether or not they were changing altitude. All aircraft held straight trajectories. Again, targets did not change from proximate to non-proximate, or vice versa, during the video.

\section{Experimental Conditions}

Between-subjects experimental conditions were the same as the Traffic Rating task: Proximate Status Indication (two levels, With Indication and Without Indication) and Traffic Density (also two levels, Low Density and High Density). For this task the Low Density condition had only two targets, specifically the lesser and greater threat converging traffic, while the High Density condition had six targets.

A within-subjects variable in this task was the Source of the Greatest Threat. The two levels for this variable were Proximate or Non-proximate. For each pilot, half of the time the Proximate target was the greatest threat and half the time the Non-proximate target was the greatest threat.

\section{Performance Measure}

Performance of the proximate status indication is represented by the proportion of pilots who correctly select the target with the greatest threat. This design indicates if the proximate status indication can interfere with assessing threat. For example, it is possible that pilots assume the proximate status indication represents a low-level alert. Thus, those in the With Indication condition will often pick the Proximate target as the greatest threat when in fact the Non-proximate target is the greatest threat, resulting in the proportion correct for Non-proximate greatest threats being lower than the proportion correct for Proximate greatest threats. Participants in the Without Indication condition would show no difference in the proportions of 
correct responses between Non-proximate and Proximate greatest threats.

It is also possible that the proximate status indication will facilitate threat assessment in these kinds of situations. Perhaps pilots cannot tell which target is the greatest threat except by the proximate status indication. Pilots in the With Indication condition would still be correct when the Proximate target is the greatest threat, while incorrect when the Non-proximate target is the greatest threat. However, pilots in the Without Indication condition would perform poorly regardless of which target was the greatest threat.

Pilots were also asked to rate their confidence in their selections of the target with the greatest threat. The confidence scale ranged from 1 for "Complete Guess" to 7 for "Absolutely Certain." It is possible that performance is poor for a condition, but pilots realize this. If performance is poor and pilots realize it, then it has no operational implications, since pilots are not expected to act on interpretations in which they have low confidence. On the other hand if performance is poor but pilots have high confidence, then it suggests that pilots may act on unreliable interpretations.

\section{Task 3: Operational Experience}

The purpose of the third task was to gather freeresponse information on how the proximate status indication is used operationally, if at all, and what value it has for pilots. This task was intended to identify any potential functions beyond those tested in Tasks 1 and 2, and to assist in interpreting the results from the other tasks. Participants were first asked the following question (bold print was present in the text seen by participants):

Based on your operational flight experience, do you feel that distinguishing traffic with $\downarrow$ and the $\diamond$ symbols on TCAS traffic displays is useful?

Participants who answered "yes" to this question were asked to "describe a situation where the distinction is useful." Participants who answered "no" were asked to "explain further, with examples if possible."

Participants were then asked the following question (bold emphasis in the original):
Similarly, based on your operational flight experience, are there any situation(s) when you felt that the distinction between $\forall$ and the $\diamond$ symbols on TCAS traffic displays caused confusion or created complications?

Participants who answered "yes" to this question were asked to "describe situations where the distinction created confusion or complications." Participants were also asked, "What changes to the two symbols above would help clear up the confusion or complications?"

Participants' open ended responses were reviewed and a categorization scheme was developed. Using this scheme, two judges independently classified the responses into six categories. Inter-judge agreement on the categories ranged from $93 \%$ to $98 \%$, and the inter-judge correlations ranged from 0.763 to 0.898 , except for the category of statements that the proximate status indication is an inadequate indication of threat, which had a correlation of 0.394 , a sentiment that was difficult to tease out of the free-response answers. The remaining categories are presented in the Results. Classification disagreements were resolved by consensus.

\section{Task 4: TCAS Logic Knowledge}

The purpose of the fourth, and last, task in the study was to evaluate pilot knowledge and understanding of the proximate status indication with eight true-false questions on what was described to the pilots as "the definitions of TCAS symbols." Four of the items compared the TA symbol (yellow circle) to the proximate symbol (filled diamond) and four compared the proximate symbol (filled diamond) to the symbol for non-proximate traffic (unfilled diamond). Among each four, the first and third items distinguished threat from non-threat symbols. For example, the items comparing the proximate to nonproximate symbols were the following:

- is always a more imminent collision threat than $\diamond$.

- always requires more prompt awareness by you than $\diamond$

The second item in each set of four distinguished one symbol from another in terms of proximity. The following item is comparing the 
proximate to non-proximate symbols (emphasis in the original):

- is always within a certain distance and altitude boundary around your own aircraft, whereas $\diamond$ is outside that boundary.

The items for TA versus proximate symbols were identical, except that TA symbols replaced proximate symbols, and proximate symbols replaced non-proximate symbols.

For each item, the percent of pilots who believed each of these statements to be true was determined. Overall symbol knowledge was measured by the percent correct score across the four TA symbol questions and across the four proximate symbol questions.

\section{Results}

\section{Task 1: Traffic Rating}

Overall, threat ratings were relatively consistent, with an $R^{2}$ of 0.47 . This is significantly higher than pilots could theoretically achieve if they only considered the proximate status $\left(R^{2}=0.18, p<\right.$ $0.0001)$ or if they only considered range $\left(R^{2}=0.30, p\right.$ $<0.0001)$ or relative speed $\left(R^{2}=0.41, p=0.0002\right)$; it is also significantly better than combining proximate status indication with a categorical representation of speed $\left(R^{2}=0.41, p=.0004\right)$.

However, a Proximate Status Indication by Traffic Density analysis of variance (ANOVA) revealed no significant effects or interactions concerning the proximate status indication (minimum $p=0.605$ ). In other words, having filled and unfilled symbols did not help pilots make threat ratings consistent with actual threat.

Overall visibility ratings were similarly consistent $\left(R^{2}=0.48\right)$, but not as high as might be expected. This value of $R^{2}$ is significantly lower than what pilots would theoretically achieve by simply classifying all proximate targets as visible and nonproximate targets as not visible $\left(R^{2}=0.59, p<\right.$ $0.0001)$

As with threat rating, a Proximate Status Indication by Traffic Density ANOVA revealed no significant effects or interactions concerning the proximate status indication (minimum $p=0.548$ ). So, symbol fill did not help pilots to be more consistent with visibility rating either.

Looking at the weights for the regression equations gives some insight into how pilots evaluate threat and visibility from a traffic display. For threat, time to CPA was decomposed into Range and Speed parameters. A mixed-design ANOVA revealed a significant main effect of parameter $(F(2.3,266.9)=$ 62.61, $p<0.001)$.

Table 4 lists the average unsigned standardized weights for each rating. For threat rating, all parameter weights are significantly different from zero, indicating that pilots are generally taking into account all parameters in their threat assessments. However, we see pilots weighing Range significantly more than Speed. This implies that while pilots are combining Range and Speed to get some approximation of time to CPA, they are overemphasizing Range. The relative weights for Lateral and Vertical Miss Distances are hard to interpret since they likely depend on the actual miss distances, which were arbitrary values in this experiment.

Table 4. Overall weights for rating traffic

\begin{tabular}{|l|l|c|}
\hline Rating & Parameter & $\begin{array}{c}\text { Standardized } \\
\text { Weight }\end{array}$ \\
\hline \multirow{5}{*}{ Threat } & Range & 0.561 \\
\cline { 2 - 3 } & Speed & $0.271^{\mathrm{a}}$ \\
\cline { 2 - 3 } & Lateral Miss Distance & $0.246^{\mathrm{a}}$ \\
\cline { 2 - 3 } & Vertical Miss Distance & 0.342 \\
\hline \multirow{3}{*}{ Visibility } & Range & 0.504 \\
\cline { 2 - 3 } & Ground & $0.093^{\mathrm{b}}$ \\
\cline { 2 - 3 } & Range by ground & 0.315 \\
\hline
\end{tabular}

${ }^{\mathrm{a}}$ Not significantly different from each other

${ }^{b}$ Not significantly different from zero.

For visibility, we see pilots weighed Range the most, but also significantly weighed the Range-byground interaction, implying they expect visibility to drop off quicker for targets below the horizon. This seems reasonably close to the true effects of ground clutter and range on visibility.

While there were no differences in the $R^{2}$ values for Traffic Density or Proximate Status Indication, the ANOVA for the weights revealed a significant Proximate Status Indication by Traffic Density by Parameter interaction for threat rating, indicating that 
the relative weight pilots place on each parameter depends jointly on the traffic density and whether they had the proximate status indication or not. The two graphs in Figure 3 show the mean unsigned standardized weights illustrating this interaction, with the top graph showing the means for Range and Speed, and the bottom graph showing the miss distances. Points connected by solid lines, including vertical lines, are significantly different from each other, while points connected with dashed lines are not significantly different from each other.
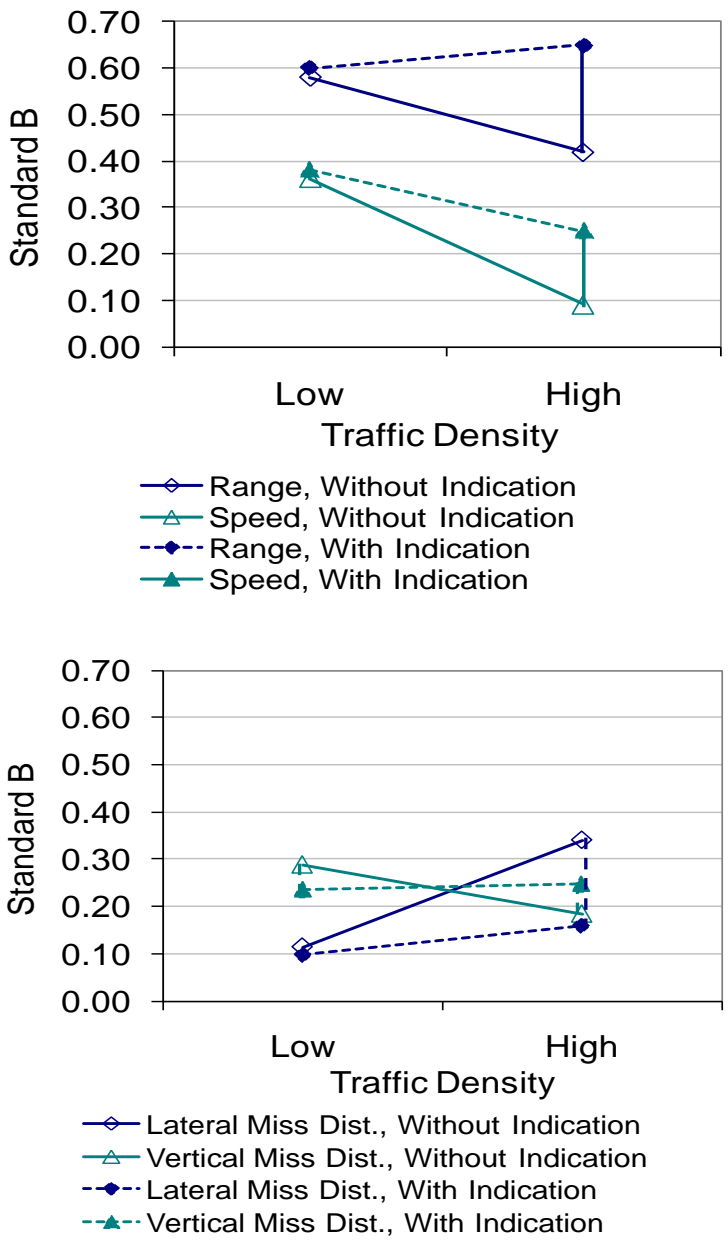

Figure 3. Mean weights for threat ratings.

The interactions and means in Figure 3 imply pilots with and without the proximate status indication apply different strategies for dealing with the increased workload imposed by higher traffic densities. With low traffic density, pilots in either Proximate Status Indication condition (With and Without Indication) used essentially the same weights for all parameters. However with high traffic density, those in the Without Indication condition, reduce weight on Range, Speed, and Vertical Miss Distance and increase weight on Lateral Miss Distance. Those in the With Indication condition, reduce weight on Speed only, and not as much as those in the Without Indication condition.

For the visibility parameters, Traffic Density and the Proximate Status Indication had no significant main effect or interaction.

\section{Task 2: Greatest Threat}

A mixed-design Proximate Status Indication by Traffic Density by Source of the Greatest Threat ANOVA found that pilots tended to correctly identify greatest-threat targets that were proximate $(92 \%$ correct on average, main effect $F(1,97)=268.1, p<$ 0.0001 ), but they were equally likely to be wrong or right in identifying greatest-threat targets that were non-proximate $(46 \%$ correct on average, not significantly different from $50 \%, t(101)=-1.56, p=$ 0.123 ). This was true whether the pilots had a proximate status indication (symbol fill) or not. Overall, pilots chose the proximate target $74 \%$ of the time, rather than the correct proportion of $50 \%$.

Whether the greatest threat was from a Proximate or Non-proximate source, pilots were on average correct $9 \%$ more often when they were in the Without Indication condition than when they were in the With Indication condition $(F(1,97)=6.403, p=$ $0.013)$. The use of fill to indicate proximity apparently interfered with accurate comparisons of traffic regardless of the proximity of the greatest threat.

There was no effect of Traffic Density ( $p=$ $0.210)$ on identifying the greatest-threat target. The interactions were not significant (smallest $p=0.204$ ).

In a Proximate Status Indication by Traffic Density by Source of the Greatest Threat by Scenario ANOVA, there were significant differences in the scenarios, where pilots tended be correct more often when lesser-threat converging targets had high time to CPA rather than high miss distances $(F(7.9,124.3)=50.94, \quad p<0.001)$. There were no interactions with Proximate Status Indication (smallest $p=0.602$ ).

Overall pilot confidence was moderate, averaging 4.30 on a $1-7$ scale, where 1 was "complete 
guess" and 7 was "absolutely certain." Pilots apparently recognized that this was a hard task, but they did not feel that they were simply guessing on average. This was true even when the non-proximate target was the greatest threat, which corresponded to performance that was in fact not significantly different from guessing. Overall there was virtually no correlation between confidence and the probability of being correct $(r=0.074)$.

However, a Proximate Status Indication by Traffic Density by Source of the Greatest Threat ANOVA on the confidence scores found a significant Traffic Density by Source of the Greatest Threat interaction $(F(1,97)=12.89, p=0.001)$, illustrated in Figure 4.

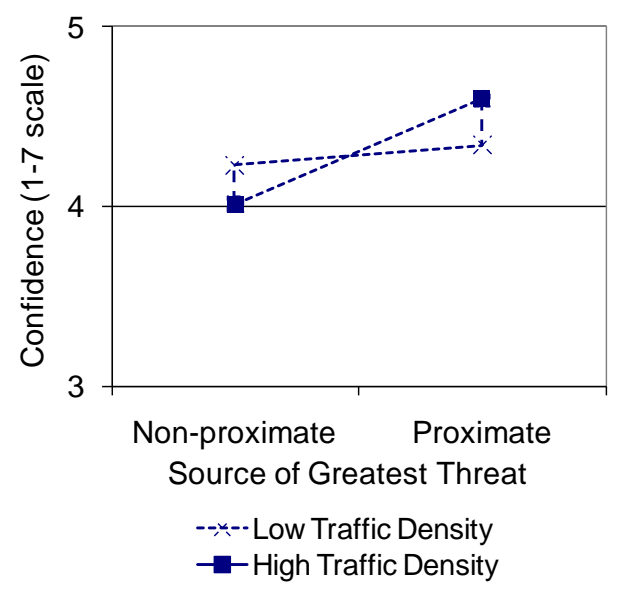

Figure 4. Mean confidence ratings.

When traffic density was high, pilots were more confident when the proximate target was the greatest threat than when the non-proximate target was the greatest threat. When density was low, there was no difference. It seems that when workload is low and pilots can study each target more carefully, pilots find it easier to convince themselves they are right about non-proximate threats or they second-guess themselves more about proximate threats.

There were no significant effects or interactions involving Proximate Status Indication (smallest $p=$ $0.454)$. There was no evidence that the proximate status indication affects pilot confidence in threat ratings.

\section{Task 3: Operational Experience}

Eighty-three percent of the pilots regarded the proximate status indication as useful. In reading and categorizing the pilots' free responses, $69 \%$ of these pilots appear to say the proximate status indication helps them prioritize their attention ${ }^{1}$. This includes $45 \%$ who specifically said it helps them attend to potential threats, conflicts, or TAs, such as this response:

$$
\begin{aligned}
& \text { It distinguishes [which] target is possibly } \\
& \text { considered a bigger threat }
\end{aligned}
$$

Eleven percent said the proximate status indication helps them with visual acquisition, such as this pilot:

\section{I... am more likely to make an effort to begin} visual acquisition

Other pilots gave no specific response, such as this one:

It helps for a quick glance to see something of note.

No one specifically said they used the proximate status indication to determine whether traffic might be visible.

Only nine out of the 101 pilots regarded the proximate status indication as potentially confusing or complicating. Of these nine, four felt more information should be incorporated into the symbol

Perhaps a short vector symbol on the intruder showing relative closure bearing.

Three of the nine stated the symbology should be changed, such as this pilot:

\section{Color coding is best}

An additional 11 pilots who did not see any problems with the proximate status indication nonetheless also suggested changing the symbology, generally to make it more obvious.

Among all the pilots, some suggested the meaning of the proximate status indication was an inadequate indication of threat, such as this pilot:

Doesn't provide a lot of trend information and can be misleading

The number of pilots who felt this way is unknown given the low reliability of this category of

\footnotetext{
${ }^{1}$ Italic text in this section represent the response categories.
} 
response. However, only four pilots were classified this way by either of the two judges who categorized the comments, so that may be an approximate upper bound.

\section{Task 4: TCAS Knowledge Results}

The Task 4 data were analyzed with a multivariate ANOVA with symbols as the independent variable (TA-versus-Proximate symbol and Proximate-versus-Non-proximate symbol), and the four items used for each as the dependent variables. Overall performance on TA-versusProximate symbol was better than Proximate-versusNon-proximate symbol (79\% correct versus $57 \%$ correct, Wilks Lambda $=0.765, F(4,94)=7.216, p<$ $0.001)$. Table 5 shows the percent of pilots agreeing with each item. A check mark next to the percent value indicates that the item was true, so pilots agreeing with that statement were correct. An X next to the percent indicates that the item was false, so pilots agreeing with that statement were incorrect.

Table 5. Pilots agreeing with each item.

\begin{tabular}{|l|c|c|}
\hline Item & $\bullet$ vs. & $\bullet$ vs. $\diamond$ \\
\hline $\begin{array}{l}\text { Is always a more imminent } \\
\text { collision threat }\end{array}$ & $85 \%$ & $65 \% \times$ \\
\hline $\begin{array}{l}\text { Always requires more } \\
\text { prompt awareness by you }\end{array}$ & $85 \%$ & $68 \% \times$ \\
\hline $\begin{array}{l}\text { Is always within a certain } \\
\text { distance and altitude }\end{array}$ & $47 \% \times$ & $64 \%$ \\
\hline $\begin{array}{l}\text { Agreeing is correct } \\
\mathbf{X} \text { Agreeing is incorrect. }\end{array}$ \\
\hline
\end{tabular}

Most participants agreed that a traffic target represented by a proximate symbol is always a more imminent collision threat, requires more prompt awareness, and is closer than a traffic target represented by a non-proximate symbol. In fact, only the last item is a correct statement about the proximate symbol. Perhaps surprisingly, about half of the pilots agreed that traffic represented by a $T A$ symbol must also be closer than traffic represented by a proximate symbol, which is not a true statement.

Performance on the knowledge questions was not related to the experimental conditions (smallest multivariate $p=0.320$ ), nor were there any significant interactions between TCAS knowledge and the Proximate Status Indication conditions. In other words, the experimental conditions did not lead to pilots misunderstanding the meanings of these TCAS symbols. Neither performance on the video tasks nor the weights in Traffic Rating tasks differed depending on pilot knowledge.

In the Greatest Threat task, participants were slightly more likely to identify greatest-threat nonproximate targets correctly if they were knowledgeable of the Proximate/Non-proximate distinction $(r=0.234, p=0.019)$. However, they were slightly less likely to correctly identify greatestthreat non-proximate targets if they were knowledgeable of the Proximate versus TA symbol distinction $(r=-0.216, p=0.030)$. Finally, knowledge of TCAS symbols is not significantly related to pilot experience variables such as flight hours, time since most recent TCAS training, or frequency of experiencing alerts (smallest $p=0.215$ ).

\section{Results Summary}

The results suggest that the proximate status indication apparently provides no performance benefit for recognizing either traffic threat or potential for visual acquisition, and it may interfere slightly with comparisons of traffic when determining which target is a more imminent threat (as defined by TCAS logic). The majority of pilots nonetheless considered the proximate status indication useful for attention allocation. They understand it represents proximity, but believe it also represents threat (i.e., a relatively greater potential for collision that requires more prompt awareness).

\section{Discussion}

In the Traffic Rating task, the presence of the proximate status indication on a traffic display did not significantly affect the consistency of traffic threat and visibility ratings with objective indications of the threat and visibility. Analysis of the weights pilots placed on the parameters of threat indicated that both groups of pilots functionally incorporated target closeness into their threat assessments. Pilots without the proximate status indication apparently did as well as pilots with the proximate status indication by observing the target's position on the display and its altitude tag.

The differences in weights associated with differences in traffic density suggest that the 
proximate status indication allows pilots to consider more parameters when under high workload. Yet this did not translate into better overall performance since there was no significant Proximate Status Indication by Traffic Density interaction for $R^{2}$. It appears that the proximate status indication may facilitate pilots' range estimates but it also overemphasizes range. This may be because pilots associate the symbol's intensification (becoming filled) with lower ranges (less than $6 \mathrm{~nm}$ ). The weight analysis indicated that pilots already overemphasize range, which may negate any advantage from the indication itself.

The Greatest Threat task found evidence that the proximate status indication may interfere slightly with comparing the threat levels of two targets. When faced with proximate and non-proximate targets, pilots tended to be less accurate in identifying the greatest threat among them when the targets were distinguished by the proximate status indication (symbol fill). This lower accuracy occurred both when the greatest threat was proximate and nonproximate. Operationally, this may result in a nonoptimal allocation of attention, such as for scanning out the window for traffic.

The Traffic Rating tasks did not reveal poorer performance for the proximate status indication when a single target was rated in isolation, suggesting that the poorer performance in Greatest Threat is specific to comparing targets. It may be perceptually more difficult to compare symbols on range or speed when they are visually different (filled and unfilled), even when the visual differences are redundant or irrelevant for range or speed estimations.

Results from the Operational Experience task indicate that most pilots consider the proximate status indication to be important for directing attention towards targets of greatest threat. Pilots completed the operational experience task after completing tasks concerning the relative threats of traffic, so it is possible that experience exaggerated the proportion of pilots who actually use the proximate status indication for threat estimation. However, this should have not diminished the frequency that other uses would also have been mentioned. It appears that the only common function pilots have found for the proximate status indication is to direct attention towards the proximate targets.

The TCAS Knowledge task indicates that most pilots are aware that a proximate symbol means a target must be relatively close, but most pilots also erroneously believe the proximate target must be a relatively greater threat that requires more prompt attention. This opens the possibility of pilots misusing the proximate status indication, although there was no evidence of such misuse in the first two experiment tasks.

Pilots tend to simultaneously regard the proximate status indication to represent both proximity and threat, which makes sense if they equate proximity with threat. Such an interpretation is consistent with the observation that about half of the pilots also think a TA symbol necessarily represents greater proximity than a proximate symbol. It is also supported by the findings from the Greatest Threat task that pilots were correct far more often when the greatest threat was a proximate rather than a non-proximate target, including when no proximate status indication was used. Finally it is supported by the weight analysis in the Traffic Rating task, which indicated that, while pilots consider other parameters in estimating threat, they overemphasize the role of proximity in threat assessment.

The tendency to overemphasize proximity in threat assessments may be a natural consequence of pilots using a plan-view display. Proximity may be more conspicuous than other parameters, such as relative closing speed. Pilots likely have observed TA symbols turning into Resolution Advisory (RA) symbols as a target draws closer, highlighting the role of closeness in threat. It seems less likely they would notice a change in target relative speed turning a TA to an RA. A pilot may imagine that, compared to a non-converging non-proximate target, a nonconverging proximate target could quickly become an imminent collision by simply maneuvering, and thus necessarily represents a greater potential threat. However, this reasoning disregards that, all other things being equal, the non-proximate target can turn onto a collision course with a smaller maneuver than the proximate target.

The possibility also exists that the proximate status indication on TCAS displays has itself encouraged pilots to overemphasize proximity in threat perceptions. In the Operational Experience task, one pilot remarked that "there is a logical progression from open diamond to solid diamond to yellow circle to red square [that] is fairly easy to understand as far as 'threat levels." Pilots understand 
correctly that the transitions from proximate symbol to TA to RA represent increases in threat. It is reasonable that they would assume the transition between non-proximate to proximate symbols would also represent an increase on the same dimension. Given that pilots generally know that symbol fill represents a change in proximity, it is reasonable to conclude that threat is primarily a matter of proximity.

\section{Experiment Limitations}

There are several limitations to this study. First, we used the TCAS algorithm as our objective definition of threat to which we compared pilot performance. There may be other measures of threat that, for example, place more emphasis on range, which pilots are inclined to do. However, the TCAS definition of threat is widely accepted and has proven effective in operational contexts. We know of no better definition of objective threat.

It has been suggested that the real value of the proximate status indication is seeing a target change from non-proximate to proximate as a cue that the traffic is closing, and therefore a threat. However, in the Operational Experience task, only a handful of the pilots alluded in any way to using the proximate status in this manner. In any case, such a use is relevant only to TCAS. CDTI has better indications, such as directionality, to indicate rapidly converging targets.

We only tested the proximate status indication for threat and visual acquisition assessments. It may have other more suitable uses. For example, it may be useful for guiding the direction of visual search, or to distinguish traffic near ownship altitude for better mental representation of traffic in three dimensions. However, no such uses were reported by pilots in the Operational Experience task. This suggests that if such uses exist then, after years of TCAS use, pilots have yet to discover them.

The web-based tasks in this study have limited operational realism. For example, traffic in the videos never turned and rarely do pilots study a traffic display for a continuous 15 second period; multiple shorter glances over a longer time would be more realistic. However, for this to be an issue, the lack of realism must be associated with a reasonable mechanism that would yield different results. For example, there should be some explanation for why a series of quick glances at a traffic display would produce an advantage for the proximate status indication that disappears with a single 15 -second gaze.

\section{Future Research Directions}

In order to determine the value of a proximate status indication any more conclusively than this study did, additional intended functions of the indication would need to be defined and evaluated. If a function for the proximate status indication is identified and a related performance benefit is documented, then the proximate status indication should be included on shared CDTI-TCAS displays.

Another direction for future research is to consider the impact of making the filled symbols consistent with pilots' interpretations. If pilots tend to think symbol fill is an intuitive indication of threat, then perhaps CDTI manufacturers should make that an actual indication of threat, basing it primarily on time to CPA, or other parameters that are not readily apparent from information on the display. For example, symbol fill could represent an "advisory" level alert [6] or an "indication" such has found in SURF-IA displays [7].

For this line of research, questions include:

- Does an "advisory" alert level "indication" improve pilot performance?

- Is it necessary to encode this "advisory" alert level or "indication" graphically in the symbol, or can this information already be adequately inferred from the target position and trajectory?

Research by Wickens and Colcombe [8] suggests that multiple alert levels do not necessarily result in better performance. How are pilots supposed to use this new alerting information? A warning such as an RA implies the pilot must commit an immediate action. A caution such as a TA implies the pilot must have immediate awareness, to get ready for an action. Is it helpful to have a sub-caution level of alert that essentially informs the pilot to get ready for getting ready to do something?

Finally, another research direction is to investigate the use of symbol fill for indicating information other than proximity or threat on CDTIs. 
This research could examine the current definition of symbol fill (i.e., target within a given altitude and range of ownship), or it could examine an alternative definition for symbol fill (e.g., traffic that is coupled, has high data quality, or is airborne rather than onground). The key research questions then become (a) How would this work on an integrated TCAS/CDTI display and (b) What issues arise if TCAS does show proximity with symbol fill while CDTI does not?

Whatever the alternative uses of symbol fill for future displays, the result of this study suggest that, given the limited visual features available in a symbol, displaying proximity graphically in the symbol is of relatively little benefit to the pilot in terms of assessing threat and potential for visual acquisition.

\section{References}

[1] RTCA, 2009, Minimum Operational Performance Standards (MOPS) for Aircraft Surveillance Applications System (ASAS), DO-317, Washington, DC, RTCA.

[2] Chandra D., Zuschlag, M., Helleberg,J., and S. Estes, 2009, Symbols for Cockpit Displays of Traffic Information. 28th Digital Avionics Systems Conference, October.

[3] RTCA, 2008, Minimum Operational Performance Standards for Traffic Alert and Collision Avoidance System II (TCAS II), DO-185B, Washington, DC, RTCA.

[4] Federal Aviation Administration, 2000, Introduction to TCAS II Version 7, Washington, DC, Federal Aviation Administration

[5] Andrews, J. W., 1991, Air-to-Air Visual Acquisition Handbook, ATC-151, Lexington, MA, Lincoln Laboratory.

[6] Warning, caution, and advisory lights, 14 Code of Federal Regulations, Pat 25, §25.1322, 1976.

[7] RTCA, 2010, Safety and Performance Requirements (SPR) for Aeronautical Information Services (AIS) and Meteorological (MET) Data Link Services, DO-324, Washington, DC, RTCA.
[8] Wickens, C., and A. Colcombe, 2007, Dual-Task Performance Consequences of Imperfect Alerting Associated with a Cockpit Display of Traffic Information, Human Factors, 49(5), pp. 839-850.

\section{Acknowledgements}

This paper was prepared by the Behavioral Safety Research and Development Division at the Volpe National Transportation Systems Center. It is based on a more comprehensive technical report in preparation. This research was completed with funding from the FAA Human Factors Research and Engineering Group (AJP-61) in support of the Aircraft Certification Service Avionics Branch (AIR130) and the Technical Programs and Continued Airworthiness Branch (AIR-120).

We thank the FAA program manager Tom McCloy and FAA technical sponsor Bill Kaliardos for their assistance. Wes Olson of the Massachusetts Institute of Technology, Lincoln Laboratory also provided valuable insights and feedback on the study. Particular thanks go to Matt Isaacs from the Volpe Center for creating the web interface used for data collection and to Andrew Kendra and Alan Yost for creating the traffic video scenarios using their CDTI simulator software. Thanks also to all the pilots who participated in the study and to the many organizations and individuals who contributed.

The views expressed herein are those of the authors and do not necessarily reflect the views of the Volpe National Transportation Systems Center, the Research and Innovative Technology Administration, or the United States Department of Transportation.

30th Digital Avionics Systems Conference October 16-20, 2011, Seattle, WA 